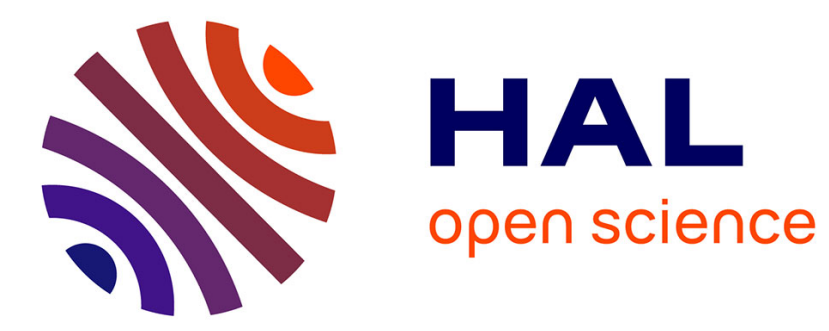

\title{
A propos de la Montagne Noire armoricaine
}

Claude Klein

\section{To cite this version:}

Claude Klein. A propos de la Montagne Noire armoricaine. Bulletin de l'Association de géographes français, 1957, 269-270, pp.22-36. 10.3406/bagf.1957.7545 . insu-02284873

\section{HAL Id: insu-02284873 \\ https://hal-insu.archives-ouvertes.fr/insu-02284873}

Submitted on 4 Mar 2021

HAL is a multi-disciplinary open access archive for the deposit and dissemination of scientific research documents, whether they are published or not. The documents may come from teaching and research institutions in France or abroad, or from public or private research centers.
L'archive ouverte pluridisciplinaire HAL, est destinée au dépôt et à la diffusion de documents scientifiques de niveau recherche, publiés ou non, émanant des établissements d'enseignement et de recherche français ou étrangers, des laboratoires publics ou privés.

\section{()ㅜ) $\Theta$}

Distributed under a Creative Commons Attribution - NoDerivatives| 4.0 International 


\section{A propos de la montagne noire armoricaine}

Cl. Klein

\section{Citer ce document / Cite this document :}

Klein Cl. A propos de la montagne noire armoricaine. In: Bulletin de l'Association de géographes français, $\mathrm{N}^{\circ} 269-270,34 \mathrm{e}$ année, Novembre-décembre 1957. pp. 22-36;

doi : https://doi.org/10.3406/bagf.1957.7545

https://www.persee.fr/doc/bagf_0004-5322_1957_num_34_269_7545

Fichier pdf généré le 25/04/2018 
Communication écrite de M. Cl. KuElN

\section{A propos de la Montagne Noire armoricaine (1)}

L'étude de la bordure méridionale de la Montagne Noire nous a conduit à examiner trois séries de problèmes :

- des problèmes stratigraphiques,

- des problèmes tectoniques,

- des problèmes morphologiques.

\section{LES pronlimes stratigaphiques}

Sur la première édition de la carte géologique de Châteaulin (1886), Ch. Barrois avait représenté, à la base de l'étage du Grès armoricain, un affleurement de schistes à clintonites (S 1 a), en observant dans la Nolice que "les banes de poudingue (du Cap de la Chève) disparaissent dans les Montagnes Noires ». L'aflleurement n'existe plus sur la carte Brest-Lorient au $1 / 320.000$ (1943), ni sur la seconde édition (fort yeu différente de la première) de la feuille de Châteaulin (1949); dans les deux cas le Grès armoricain repose dircctement sur les schistes briovériens.

1"La base de la série paléozoüque. - Nos recherches dans la région nous permettent d'affirmer que la série paléozoïque débute constamment par un magnifique poudingue a galets de quart\% et a ciment siliceux ou schisteux. On le suit sans difficulté clans tout l'intervalle compris entre Kervivic à l'Est et la cluse du Ménie à l'Ouest. On l'observe notamment à Kergrech ; entre Kergrist et le Roc de la Madeleine ; au Nord de Guernach et de Kersaliou; au Clunbras ; au Nord de Goastom et de Garsdarid ; a Coat Quilvern, entre Mendy et Roctoul, au Nord de Kergoat; au Grand Plessis, a Kervasor, au Sud du Ménie...

Au triple point de vue de la nalure des galets el du ciment, du calibre et de la forme des galets, on ne seurait le distinguer du poudingue que nous avons rencontré a la base de la série pourprée dans les synclinaux de Réminiac et de Poligné ou au long du bord Nord du synclinal de St-Julien-de-Vouvantes entre la cluse de l'oust et celle de la Vilaine (feuille de kedon).

Ce poudingue est surmonté lantôt par des schisles violels en dalles, tantót par le Grès armoricain, tantôt directement par les schistes ardoisiers à Calymènes. Les schistes ( $S$, ou $\mathrm{S} 1$ a des cartes). de teinte ruse violacée ou violette, sont visibles au Nord de Kergrist

(1) Les chifres en caracteres gras renvoient a la bibliographie placéc a la fin de l'article. Les references a la these de M. Grandals sont indiquées dans le texte sous lit forme : These, p. .. 
ou de Trévénou, à Kersaliou, au Clunbras, à Coat plin Coat, à Roctoul, au Grand Plessis. L'assise, généralement mince, mais bien identifiable, peut disparaître et le poudingue être surmonté par le grès : ainsi au Nord de Keransker.

C'est l'assise de ce grès-quartzite qui forme le sommet de la longue crête méridionale de la Montagne Noire. La carte topographique montre trois sections dans lesquelles la crête présente une largeur maxima : au Nord de Trégourez, au Nord de Roudouallec, dans la forêt de Conveau. Par contre, dans les sections intermédiaires, la crête se morcelle en un chapelet de buttes étroites et allongées, diversement orientées (de Ouest-Sud-Ouest-Est-NordEst à Ouest-Nord-Ouest-Est-Sud-Est).

Cette disposition exprime les données immédiates de la structure. L'assise du Grès armoricain n'a pas, en effet, la belle régularité de puissance et de faciès que lui assigne la carte (1). 'Tout au contraire on assiste fréquemment à des réductions d'épaisseur qui peuvent aller jusqu'à l'effaccment complet du faciès grès. On voit alors les schistes violets en contact direct avec les schistes noirs (S 2 a) (à Roctoul, bien que l'existence d'un décrochement Nord-Est-Sud-Ouest ne permette pas d'être catégorique). Au Nord de Goastom, entre le point coté 181 et la butte cotée 252 on assiste même à la superposition stratigraphıque du loudinģue et des schistes à Calymènes. Le passage du poudingue - à ciment schisteux noir - aux schistes ardoisiers est progressif et l'hypothèse d'une juxtaposition d'origine mécanique (11) ne peut être retenue. Ces variations latérales de puissance et de faciès s'observent souvent au voisinage des petits décrochements transversaux que les rivières ont utilisés et qui découpent l'arête Sud de la Montagne orientée Nord $75^{\circ} 80^{\circ}$ Est, en une série de petits éléments Est-Ouest. Entre Mur de Bretagne et Uzel (feuille de Pontivy) nous avons observé des faits du même ordre qui reflètent la diversité des circonstances sédimentologiques.

G'est donc, en dernière analyse, l'assise des schistes d'Anger's qui serait la plus constante avec celle du Poudingue. On est alors fondé à estimer qu'ici, comme au Sud de Rennes, la série paléozoïque débute à l'Ordovicien - vraisemblablement à l'Arénig (2) - et que le poudingue à galets de quartz en constitue la base.

$2^{\circ}$ Le poudingue de Gourin pose un second problème stratigraphique. La deuxième ćdition de la feuille de Châteaulin représente deux alignements principaux de poudingue dits « de Gourin 》.

(1) ...et qui a permis à $M$. Guilcher d'écrire : «Le travail d'amenuisement a eu lieu également aux dépens de la crête de Grès armoricain, qui elle aussi n'occupe qu'une partie de l'affleurement... »Thèse, p. 32.

(2) C'est-à-dire à un niveau stratigraphiquement un peu plus élevé qu'au $S$. de Rennes où les schistes de la Série pourprée représenteraient le Trémadoc. Le Cambrien, de toutes manières, ne serait représenté ni ici, ni là. 
- l'un va de Sabar Hellen à Kéransquer, par le Queidel, au Nord de Roudouallec;

- l'autre de Gourin à Kerbescontez en suivant la route de Plouray.

La notice présente ce poudingue comme interstratifié au sein du Briovérien (étage $X^{b}$ ) et nous savons que Ch. Barrois et $P$. Pruvost l'ont considéré comme représentant en Bretagne la base du Cambrien, c'est-à-dire comme équivalent latéral du poudingue des Coëvrons et du poudingue cie Rocreux (1). D'autres auteurs n'y voient qu'un poudingue intraformationnel briovérien (précambrien ou infracambrien). Rappelons que Dufrénoy le considérait comme représentant la base du Silurien discordant sur les schistes et phyllades de St-Lò plus anciens (8-p. 251-252).

Nos observations dans la région de Gourin et de Roudouallec nous ont montré une distribution beaucoup plus large des affleurements de ce poudingue à galets de quartz. Il constitue, notamment à l'Est de Gourin, une série de placages nappant des buttes ou des croupes et on peut le suivre de Cran Pipidic jusqu'à la butte cotée 229 (au Sud de la Forêt de Conveau) par la butte 178, Brunot, Kerguicher, Kérendilly, St-Nicolas, la cote 153. Ces placages sont ordonnés suivant un axe rigoureusement parallèle au talus de la Montagne Ncire. Une seconde série d'affleurements est axée sur la route Gourin-Plouray, tandis que la croupe qui unit Disméon à Kerbescontez est tout entière nappée par le poudingue et que de petits placages épars apparaissent à Guerveur-Christ, à Crondal ou encore, au Sud de Kergaradec, autour des Loges Menez lann. De part et d'autre de Roudouallec on l'observe bien à Lesvéguen où il nappe une longue croupe d'interfluve ainsi qu'au Queidel et à Kéransquer, dans la même position. C'est encore lui qui « chapeaute» la butte cotée 198 à Quistinit, signalée par Hébert (14-p. 762).

Ce poudingue à galets de quartz et à ciment siliceux, tantôt sain et massif, tantôt altéré est très souvent teinté de rouge violacé et se rencontre en association avec des grès rosés et des schistes rose violacé bien observables dans les carrières (Lesvéguen, Disméon), à l'Est de Gourin, entre Landevec et Parc Harnec ou bien à Kérendilly. Dans le triangle Kerguicher-Parc Harnec-Cran Pipidic (et sauf dans cette dernière localité), il est patent que le poudingue qui nappe les buttes n'affleure pas au fond des vallées ouvertes dans le X. A Disméon le pendage des bancs de grès rose interstratifiés n'est que de $10 \grave{a} 15^{\circ}$.

Les exigences de la figuration cartographique, la constance d'une assoriation de faciès originale (poudingue-grès rosés-schistes violacés), les similitudes pétrographiques, granulométriques et morphoscopiques entre ce «poudingue de Gourin 》 et le poudingue à galets de quartz que nous avons rencontré au bas dı talus de la Montagne Noire en association avec des schistes 
rose violacé ou violet, nous incitent à considérer que nous sommes en présence d'une seule et même formation de poudingue, l'assise de base de la série ordovicienne. Nous revenons ainsi à l'hypothèse formulée par Dufrénoy dès 1838 et acceptée par Hébert (14-p. 762-764) (3).

Le fait que nous ayons rencontré quelques cas où le poudingue paraît interstratifié dans le Briovérien (Sud de Ploermel, le Tertre au Sud de Carentoir) ne saurait servir de fondement à une règle qui, par ailleurs, se révélerait géométriquement incapable de rendre compte de la distribution de la quasi-totalité des affleurements de ce poudingue. Nous interprétons certains au moins de ces cas exceptionnels comme des intercalations d'origine mécanique, souscrivant ainsi pleinement à une remarque d'A. Bigot (2-p. 117. 119). Et nous pensons que le poudingue de Gourin constitue la formation de base de la série ordovicienne en bretagne - comme d'ailleurs en Vendée, où le poudingue de Vihiers présente le faciès Gourin. Mais il est hors de doute qu'on rencontre en Armorique, dans le Maine en particulier, un poudingue à galets de quartz (Parennes, Courmenant, la Crousille) ayant le « faciès Gourin * et dans lequel on a signalé en outre des galets de granite : ce poudingue pourrait être d'origine intraformationnelle et d'àge brioverien. En tout cas il ne saurait être confondu avec le « poudingue de Gourin » des feuilles de Redon et de Chàteaulin (16).

\section{LES PROBLìmes TECTONIQUES}

En désaccord avec $M$. Giot sur la nature, la position et la signification tectonique du poudingue de Gourin (10-11-12) nous sommes par contre tout disposé à reconnaître avec lui qu'il y a * disharmonie complète » entre le Briovérien et le Paléozoïque (12-p. 4). Elle apparaît fort bien à l'Ouest de la terminaison périsynclinoriale du bassin de Châteaulin : les schistes $\mathrm{X}$ de la baie de Douarnenez ont une orientation voisine de Est-Ouest tandis que, de Telgruc au Roch Veur, la couverture affleure suivant une direction générale Nord-Ouest-Sud-Est. En l'absence des critères paléontologiques qui permettraient de chiffrer l'importance du hiatus stratigraphique entre le Briovérien et la base de l'Ordovicien, ce désaccord tectonique vient appuyer l'idée d'une discordance majeure entre le $X$ et la couverture paléozoïque. Cette discordance ne peut être constatée directement au Sud de la Montagne Noire dont les bancs sont redressés parfois jusqu’à la verticale.

Il faut cependant définir plus précisément les rapports entre la

(3) Les faits sont d'ailleurs beaucoup plus suggestifs au long du flanc $N$ du syuclinal de St-Julien-de-Vouvantes, à l'E. de Sixt-sur-Aff comme entre la Boixière-Launay et St-Jean-des-Bois où on saisit l'évidente continuité stratigraphique d'une formation cartographice pour partie sous le signe du poudingue de Montfort, pour partie sous celui du poudingue de Gourin. 
lectonique du socle schisteux et celle de sa couverture. A cet igard nous ne pouvons admettre cette proposition de M. Giot : le Palézoïque «a donc subi une tectonique de revêtement qui n'a nullement influencé son substratum »(10-p. 181 et 12-p. 4). $11 \mathrm{y}$ a, à notre sens, des rapports précis entre les déformations du socle schisteux el celles de sa couverture discordante qui aurait exprimé en un style original les réactions propres du socle (15-16).

1" La trame directionnelle de la Bretagne occidentale fait apparaître trois orientations privilégiées :

a) Une orientation Nord $100-105^{\circ}$ Est : c'est celle de la bande granitique des Landes de Lanvaux et de l'anticlinorium au cœur duquel affleurent les arkoses de Bains. C'est celle des anticlinaux of synclinaux de la couverture paléozoïque de part et d'autre de l'ixe Lanvaux-Candé (voir la carte).

Au Nord de la Guerche-de-Bretagne, comme au sud de P'ouancé, les schistes $\mathrm{X}$ eux-mêmes ont cette direction Nord 100-105" Est et l'accord est remarquable entre le socle et sa (ouverture.

b) Une orientation Nord 115-125' Est : c'est celle des mighmalites et des granites d'anatexis de « l'anticlinal de Cornouaille » (ies géologues (5-p. 798-799), celle de la bande granulitique Pointe du Raz-Nantes, celle de la longue cicatrice mylonitisée (rhyolites ou hälleflintä des cartes géologiques) (6), celle encore clu grand filon de quartz Baie des Trépassés-Sillon de Bretagne.

c) Une orientation Nord $75-80^{\circ}$ Est : c'est la direction dite « du Léon » admirablement soulignée par la crête Sud de la Montagne Noire.

Ces trois directions paraissent converger vers le Cap Sizun. En réalité, elles se recoupent : J. Cogné l'a fort bien démontré en ce qui concerne l'anticlinal de Cornouaille et l'axe des granites de Lanvaux (5-p. 794). Il est clair d'autre part que le flanc Sud de la Montagne Noire fait avec ce mème anticlinal de Cornouaille un angle de $25^{\circ}$. Quant aux apophyses granulitiques (dont on s'accorde à admettre la mise en place tardive et qui, de ce fait, ont pu exprimer certains traits du dispositif structural) elles paraissent se détacher tangentiellement au long du flanc Nord de la grande bande granulitique Pointe du Raz-Nantes :

- au Nord de l'axe de Lanvaux ces apophyses semblent avoir obéi à la direction du Léon (granulite de Locronan, de Guelven, de Guéméné, de Pontivy, de Gucihenno et de Plumelec);

-- au Sud de l'axe de Lanvaux les apophyses soulignent la direction Nord 100-105" Est (granulite d'Allaire, du Temple-deBretagne).

Tout se passe comme si la bande granulitique Pointe du Raz-Nantes avait recoupé obliquement une trame structurale dans laquelle deux directions étaient déjà vigoureusement affirmées 
(direction «Léon » au Nord, direction « Lanvaux 》 au Sud) qui auraient orienté la mise en place des apophyses granulitiques greffées sur la bande principale.

$2^{\circ}$ Essai d'interprétation:

Les deux directions tectoniques fondamentales de l'Armorique (Léon et Lanvaux) font entre elles un angle variant de $20-30^{\circ}$ a l'Ouest à $30-45^{\circ}$ plus à l'Est. Il ne nous semble pas qu'elles aient pu apparaître simultanément au cours de l'orogénie hercynienne comme on l'admet très généralement.

a) La trame prévarisque. - Le fait que, entre l'Afr et la Mayenne, nous ayons très souvent constaté un accord directionnel entre le Briovérien et sa couverture (fort loin en avant de celle-ci) nou s conduit à penser que le socle pré-ordovicion avait été plissé suivant la direction de Lanvaux (Nord 100-105 Est passant, en Anjou, à la direction Nord $115-120^{\circ}$ Est), et que les plis du socle tronqués et masqués par la couverture paléozoïque ont simplement rejoué à l'époque hercynienne, déterminant l'apparition dans la couverture d'un système de plis de même orientation et d'amplitude modérée (16).

Le granite de Lanvaux (9), les migmatites du Belon (4) - ou granite de Moëlan (5) - certaines ectinites de l'anticlinal de Cornouaille (5-p. 793-94) présentent cette orientation. Mlle A. Faure-Muret a attribué un âge cadomien au granite de Lanvaux, âge contesté par M. Demay (7) mais accepté par J. Cogné. La discussion de cet intéressant problème sortirait du cadre de cei article, mais nous retiendrons, à titre d'hypothèse de travail, que cette orientation Nord $100-105^{\circ}$ Est et cette granitisation nous paraissent effectivement issues de l'orogénie cadomienne, admise en Normandie et qui, à notre sens, aurait intéressé l'ensemble de la France de l'Ouest.

b) L'effet des contraintes hercyniennes. - C'est sur cette trame plissée et modérément indurée que se seraient exercées les contraintes hercyniennes. La direction varisque (ou du Léon) s'est imposée souverainement de part et d'autre du bassin de Châteaulin, en Montagne Noire et dans les Monts d'Arrée. Il est d'ailleurs probable que cette direction s'est affirmée très tôt, peut-être dès l'Ordovicien (embryotectonique hercynienne) et qu'une puissante flexure séparait alors le fond subsident du bassin de Châteaulin d'une aire continentale ou épicontinentaie située au Sud et à l'Est. C'est au droit de cette flexure que les variations de puissance et de faciès des diverses assises semblent avoir été les plus fréquentes. On comprend que la barre rigide de la Montagne Noire alignée sur le tracé de cette ancienne flcxure sépare, aujourd'hui encore, deux régions gravimétriquement très différentes entre lesquelles on observe un gradient moyen de 12 à 15 milligals. 
Ccpendant si la direction du Léon, bien datée, s'est imposée au Nord d'une ligne allant du Raz à Dinan par Mur de Bretagne et si les complexes cristallophylliens et les batholites de granite ou de granulite associés obćissent dans l'ensemble à cette orientation, on a remarqué depuis longtemps une série de décrochements obliques, de déchirures au long du flane des plis de couverture qui, nous scmble-t-il, expriment la réaction du matériel l’aléozö̈que à l'interférence des directions cadomiennes héritées et des contraintes varisques.

1. La Montagne Noire est ainsi « débitée en tronçons 》 (11) d'orientation équatoriale ou subéquatoriale, parfois même Nord 95-100 Est. Nombreux parmi ces décrochements sont ceux associès à des lieux de faublesse mécanique [réduction ou disparition du faciès grès-quartzite ( $S 1$ b) l. Les circonstances sédimentologiiues justifient donc certaines localisations mais elles n'expliquent pas l'origine des cassures, qui nous paraît devoir être rechcrchée dans l'anisotropie mécanique du socle briovérien (cadomien) vis-à-vis des sollicitations hercyniennes. Il s'agirait en somme d'un jeu de tectoniques superposées (20). La direction varisque s'est imposée dans le dessin général des plis tandis que la direction cadomienne (voisine de Est-Ouest) expliquerait les particularités du détail.

L'interprétation ne vaut cependant pas pour les grands décrochements (le Grand Plessis au Sud de Laz, Roctoul au NordOuest de Roudouallec, Guernach au Nord-Est de Gourin) : l'existence de failles de cisaillement ayant intéressé à la fois le socle et sa couverture et orienté les axes principaux du réseau hydrographique est difficilement contestable. «L'accident》 de Briec et la « faille de Gourin »'en sont que les deux expressions les plus remarquables (cf. carte).

2. Dans la région silurienne comprise entre Rennes et Redon, là où les directions cadoniiennes du socle ont été réactivées à l'époque hercynienne on retrouve ces mêmes décrochements transversaux aménagés en cluses par les rivières. Leur direction la plus commune (Nord $30^{\circ}$ Ouest) est sensiblement perpendiculaire à la direction du Léon et paraît se situer dans le plan des contraintes varisques (faille de Malestroit, cluse de l'Oust, cluse de l'Aff, faille Guer-Renac). Ces failles sont sensiblement parallèles au grand accident finistérien connu sous le nom de « faille Kerforne 》 et dont le tracé va de la presqu'île de Crozon à la baie de la Forêt, par Douarnenez. Les failles minéralisées par le quartz de Plogastel-St-Germain et de Caudan (à l'Ouest d'Hennebont) ont la même orientation.

Ainsi deux trames paraissent superposées :

- la trame des plis souples dans laquelle on roit interférer 
les deux directions de Lanvaux (d'âge cadomien ?) et du Léon (d'âge hercynien);

- la trame des failles dont les directions sont orthogonales ou obliques par rapport à celles des plis précédents.

Sans vouloir prétendre tout expliquer par des considérations de géométrie tectonique dont nous n'ignorons pas les dangers, il nous semble que certaines données de l'observation s'éclairent convenablement lorsqu'on admet la coupure stratigraphique fondamentale entre la série paléozoïque et le Briovérien d'une part, l'existence de tectoniques superposées d'autre part. C'est la trame héritée de ce riche passé qui rejouera aux époques ultérieures et singulièrement au Tertiaire. Des directions tectoniques nouvelle's ont peut-être apparu, mais il est très probable que les directions anciennes mal cicatrisées ont continué à joucr modérément, au cours des temps post-hercyniens remettant souvent en cause des équilibres morphologiques lentement élaborés.

\section{LES PROBLÈMES MORPHOLOGIQUeS}

Dans sa thèse (13), M. Guilcher a présenté les deux lignes de crête de la Montagne Noire comme un relief résiduel s'enlevant au-dessus d'une surface d'aplanissement dont l'âge serait fixé par les restes d'une mince croûte de grès à Sabals : la pénéplainc éocène. « A l'Eocène, écrit-il, la Montagne Noire devait avoir l'allure d'un long Inselberg » (Thèse, p. 33). Cette conception des rapports morphologiques de la Montagne et du glacis cornouaillais, de la nature et de l'âge de ce glacis, s'appuie sur des arguments qui ont pu paraître décisifs.

- La réalité morphologique du glacis est absolument incontestable, et, malgré le jeu ou le rejeu de blocs faillés qui en ont parfois rompu la régularité, il ne fait pas de doute que nous sommes en présence d'une surface d'aplanissement qui paraît venir buter au pied de la crête du Grès armoricain dont la base même serait tronquée.

- Sur ce glacis on observe des dépôts, soit à l'intérieur du triangle Roudouallec-Coray-Guiscriff, soit entre Langonnet et Plouray. La notice de la carte de Châteaulin $\left(1^{{ }^{\circ}}\right.$ et $2^{\circ}$ éd.) nous présente ces dépôts $\mathbf{P b}$ comme "des galets de quartz blanc, jaunis, de 2 à $3 \mathrm{~cm}$. de diamètre, épars à la surface du sol, parfois cimentés par de la limonite et atteignant rarement un mètre d'épaisseur $\gg$. Cette attribution au Pliocène a été contestée par M. Y. Millon (18) pour qui « ces formations doivent être rapportées à l'Eocène », car "elles présentent des faciès comparables à ceux des grès à Sabalites de l'Ouest... ». Ce point de vue, accepté par M. Guilcher - pour qui les dépôts sont en place - lui permettait, très logiquement, "d'évoquer au-dessus de la pénéplaine éocène en voie d'achèvement, un paysage d'Inselberg, de monadnocks aux flancs abrupts $\gg(T h e ́ s e$, p. 34). 
Les observations que nous avons recueillies nous permettent cependant de préciser ou de nuancer certaines données du problème.

\section{$1^{\circ}$ Définition du versant Sud de la Montagne Noire :}

a) C'est un talus que l'œuvre de l'érosion différentielle suffit le plus souvent à expliquer. Il ne s'agit en aucune sorte d'un talus d'érosion sculpté dans la masse des grès-quartzites $\mathrm{S} 1 \mathrm{~b}$, comme la seule inspection de la carte géologique le suggère en effet. Gràce aux affleurements du poudingue à galets de quartz nous arons pu suivre la trace de la base de l'Ordovicien : elle coïncide, très généralement, avec la base du talus de la Montagne. Cette disposition est particulièrement apparente là même où la prétendue réduction d'épaisseur de l'assise du grès, imputće au recul du versant, aurait atteint ses valeurs maxima : entre le Clunbras et Coat Quilvern un admirable glacis taillé dans le Briovérien et se tenant vers $180-190 \mathrm{~m}$. est dominé par un talus au flane duquel le poudingue affleure largement parmi la lande. Aucune contestation n'est possible : le chemin creux de Goastom à la cote 181 est taillé dans la masse des schistes $\mathrm{X}$ fortement plissés et recouverts d'une mince pellicule de solifluction d'où pointent d'énormes blocs de poudingue détachés de la falaise. Au Nord de Kéransquer on peut faire la mème observation ainsi qu'au Sud de la Forêt de Conveau (à Kergrist et à Kergrech). Parfois l'assise du poudingue (d'ailleurs mince) et celle des schistes violets sont tranchées ; c'est alors la barre du grès qui s'enlève au-dessus du glacis. Mais il est clair que, dans tous les cas, nous sommes en présence d'un talus structural, du flanc externe d'une barre, dégagé par l'érosion différentielle qui a aisément déblayé les schistes briovériens au contact des assises résistantes et fortement redressćes de l'Ordovicien. (Les très nombreuses carrières ouvertes dans le grès $S \mathbf{1}$ b permettent d'affirmer que les pendages oscillent entre 65 et $90^{\circ}$ avec parfois même renversement de la séric).

On ne saurait done tirer argument du « travail d'amenuisement... de la crête de Grès armoricain... » pour en inférer la réalité d'un versant d'érosion, d'un versant d'Inselberg s'enlevant al1-r!essus d'un glacis qui tronquerait au même niveau les schisles hriovériens et la base du grès ordovicien.

b) L'origine tectonique de certains éléments du talus est extrèmenient probable.

M. Guilcher a admis que les cuvettes de Guiscriff et de Plouray sont d'origine tectonicue et cette interprétation n'est guère contestable. Le rejeu de la trame losangulaire des failles de cisaillement que nous avons évoquées plus haut (et dont le horst de Coadri ne représente qu'un élément) paraît responsable, directemenl. de la ceinture de hauteurs qui limitent, an Sud-Ouest el it 
l'Ouest le bassin de l'Isole supérieure. Deux axes auraient rejoué : - un axe Laz-Scaer jalonné par la cluse de décrochement clu Grand Plessis et par les cours de la Ster Rondou et de l'Isole ;

- un axe Roudouallec-Coray qui intéresserait la Montagne Noire à la cluse de Coat Quilvern ou à celle de Placeguen (4). Le bassin de Plouray lui-même semble limité au Sud-Est par un voussoir, dont l'axe serait parallèle au cours de l'Ellé et au Sud-Ouest par un accident allanl du décrochement du Clunbras (Nord-Ouest de Gourin) à la confluence de l'Ellé et du ruisseau de l'Etang de Langonnet.

Dans cette optique du rejeu vertical de certaines sections de la trame tectonique héritée on comprend mieux l'ordonnancement des horsts et des cuvettes. A notre sens, le petit pointement granulitique de Kergus serait un horst à l'intersection de la « faille de Gourin 》 et de la ligne de faiblesse jalonnée par la Ster Laer. Quant au relatif perchement des bassins de l'Isole et de l'Ellé il s'explique parfaitement par la difficulté qu'ont rencontrée ces deux rivières à inciser les larges bandes granulitiques qui bar. rent les deux cuvettes vers le Sud.

Il nous paraît impossible de limiter au seul glacis cornouaillais; les effets du rejeu de la trame des failles dont nous savons qu'elles intéressent à la fois le glacis et la Montagne. L'intérêt morphologique des considérations tectoniques développées plus haut nous apparaît alors et nous pensons que certains éléments du talus méridional de la Montagne Noire doivent leur vigueur et leur fraîcheur à de tels rejeux. C. Vallaux avait déjà remarqué que les points hauts compris entre Guiscriff, Roudouallec et Coray sont " à une altitude presque égale à celle de la Montagne Noire 》 (19-p. 212) et que cette région apparaît comme un véritable centre de dispersion hydrographique. L'idée d'une surélévation tectonique de la Montagne, au Nord de Roudouallec et dans la Forêt de Conveau, est donc très vraisemblable.

Le talus Sud de la Montagne serait ainsi, dans son ensemble, une forme structurale dont les caractères s'expliquent soit par lat valeur des contrastes lithologiques entre les schistes briovériens et les assises résistantes de la base de l'Ordovicien, soit par des rejeux tectoniques récents de la trame des failles par laquelle nous avons expliqué les grands décrochements qui morcellent la barre du Grès armoricain (5).

La date récente de ces rejeux expliquerait que nous soyons en

(1) Cet axe est parallèle à celui jalonné par l'Odet supérieur et par la cluse de Mendy, lui-même parallèle à la grande faille occidentale de « l'accident de Briec $\gg$.

(5) Il est d'autre part extrêmement probable que cette ligne de faiblesse mécanique qu'est la flexure liminaire $S$. du bassin de Châteaulin a rejoue lors de chaque épisode de réajustement isostatique : la distribution des anomalies gravimétriques de part et d'autre de cette ligne est, à cet érard, fort suggestive. 
présence d'une ébauche de relief appalachien (17-p. 231) et que la dépression médiane associée aux schistes ordoviciens et gothlandiens soit si mal dégagée et perchée de 50 à $80 \mathrm{~m}$. " au-dessus des vallées et plateaux extérieurs $\gg(T h e ̀ s e, p .34)$. Cette disposition s'accorde mal avec l'idée d'un relief résiduel évoluant depuis les temps prééocènes. Comment d'ailleurs justifier cette étonnante persistance d'un relief dont il ne faut pas surestimer le volume et dont l'armature rigide, souvent fort mince, n'oppose à l'érosion régressive qu'une ligne de résistance d'autant moins efficace qu'elle n'était pas continue ?

Cette jeunesse d'un élément de relief, considéré comme l'un des plus anciens de l'Armorique, nous semble mieux en accord avec les données de fait que l'hypothèse d'un Inselberg dressé à la surface de la pénéplaine éocène.

\section{$2^{\circ}$ La datation du glacis cornouaillais :}

L'assimilation par $\mathrm{M}$. Milon des dépôts $\mathrm{Pb}$ de la carte géologique aux grès à Sabalites de l'Anjou, du Maine et de Toulven - qui, eux, sont fossilifères - repose, nous le savons, sur une simple analogie de faciès. Il faut d'ailleurs convenir qu'il s'agit d'un faciès assez caractéristique pour que l'opération soit légitime malgré les risques qu'elle comporte. M. Guilcher a signalé, dans sa thèse, de nombreux témoins de ces grès. Ce n'est donc pas leur existence qui est en cause, pas même leur « âge», bien qu'à cet égard un doute subsiste, ainsi que $M$. Guilcher le reconnaît (Thèse, p. 536-537). C'est la position de ces grès qui justifie des réserves tandis que la présence, non moins incontestable, de galets roulés parmi les formations $\mathrm{Pb}$ pose le problème de cette énigmatique association.

a) Les dépôts qui tapissent le fond des cuvettes de Guiscriff et de Plouray sont-ils partout en place?

M. Guilcher le pense ct tire argument de cette position pour dater sa pénéplaine et pour chiffrer l'amplitude des déformations qui l'ont affectée. Ces conclusions seraient inattaquables si nous nous trouvions en présence, comme en certains points de Bretagne, d'une croûte siliceuse continue ou tout au moins "d'énormes blocs de plusieurs centaines de kilos $\gg(3$, p. 136), dont la position in situ est assurée. Mais à Plouray comme à Guiscriff il ne s'agit que d'éléments de 5 à $40 \mathrm{~cm}$. enrolés "dans des argiles blanches ou panachées..., parfois brunes ou rouges 》 (Thèse, p. 75) et associés « à des grès ferrugineux et à des brèches quartzeuses à ciment de limonite »(18-p. 1361).

On imagine aisément de quelle mobilité peut ètre douée une telle formation lorsqu'elle repose sur une pente, même faible, dès que les argiles matricielles sont saturées en eau.

Nous pensons donc que les dépôts en question - parfois en 
place ou «presque en place», notamment au sommet des horsts -.. et en particulier celui du Coadri - ont glissé des hauteurs qui ceinturent la cuvette au Sud et au Sud-Ouest ou de la Montagne Noire au Nord. Ils ont tapissé le fond de la dépression tectonique et moulé les formes du relief ainsi que les escarpements de faille et les flexures marginales elles-mêmes. L'opération géométrique qui consiste à prolonger le plancher de la curette jusqu'au pied du versant de la Montagne Noire et a assigner a cette surface l'àge des dépôts qui la masquent nous parât contestable : la surface en question s'incline du Sud vers le Nord en direction de la Montagne Noire et la région de Roudialaller est de 20 a $30 \mathrm{~m}$. en contre-bas du niveau moyen de la cuvette. Beaucoup plus légitime nous parâ̂t être le point de rue soutenu ha Em. de Martonne (17) et suivant lequel le relief actuel dériverail d'une surface langente au sommel des horsts el a la ligne de faîte de la Montagne, altimctriquement concordanis. il at probable, d'ailleurs, que certains éléments às curelles appartionneni a la surface des grès à Sabals, mais il est non moins assuré que les mouvements tectoniques qui ont déformé celte surface onl mompu les rapports fonctionnels qui auraient pu exister à l'ápoque éocène entre le glacis ot la Montagne. D'autre part les placages du poudingue de Gourin qui nappe de nombreuses croupes d'interfluves, dans la région de Roudouallec et à l'Est de Gourin, expliquent convenablement leur conservation.

Nous admettons donc que le glacis cornouaillais dérive d'une surface d'aplanissement longtemps et lentement regradéc qu'on peut rapporter à l'Eogène (surface de regradation lente). Mais nous pensons que cette surface nivelait la Montagne Noire ellemême et que les mouvements tectoniques qui ont déformé le glacis ont affecté en même temps et au même degré la Montagne. La pellicule des grès « à Sabals », qui avait placé «sous scellés» la topographie éogène, a été largement démantelée et remaniéc et ses débris se sont rasscmblés dans certaines cuvettes tectoniques où ils voisinent aujourd'hui avec des formations toutes différentes: les galets et poudingues « pliocènes».

b) La nature, l'âge et la signification des galets roulés associés aux grès d̀ Sabals.

M. Guilcher a reconnu que la présence de galets roulés, formés aux dépens des grès lustrés « à Sabals 》 et «présentant des dyssymétries nettemient marines » (Thèse, p. 181-183) posaient un problème délicat. Il a observé ces galets, au Nord de Toulven, jusqu'à l'altitude $+54 \mathrm{~m}$. en superposition ou en juxtaposition avec des grès à Sabals «en place » bien conservés dans la curette de Toulven elle-même.

Or de nombreux observateurs ont signalé - et nous avons personnellement reconnu, à l'Est et au Sud-Est de Leuhan - des galets emballés dans les argiles blanches ou panachées de la cuvette

5. 
de Guiscriff. S'agit-il, comme il est parfois vraisemblable, de galets de quartz issus du démantèlement des placages du poudingue de Gourin et dispersés au sein des formations $\mathrm{Pb}$ que nous venons de considérer comme le produil d'un remaniement? Ou bien s'agit-il de galets de quartzite ayant mème signification que les galets de Toulven ? Nous pensons que des galets de l'une et l'autre origine sont associés dans ces formations car on rencontre, dans une région voisine (au Nord de Pontivy et à l'Est de Loudéac, notamment autour de Merdrignac et de la Chèze) des galets de quartz ou de quartzite, parfois agglomérés en un poudingue ì ciment ferrugineux qui ne sont pas sans rappeler « ces galets de quartz blanc..., parfois cimentés par de la limonite », évoqués par Ch. Barrois dans la notice des deux éditions de la feuille de Châteaulin. Ces galets et ces poudingues s'observent au Nord-Est de la Chèze jusqu'au voisinage de $200 \mathrm{~m}$., c'est-à-dire au niveau même des curettes de Guiscriff et de Plouray. Leur âge est indéterminé : les cartes géologiques les rapportent au Pliocène. Mais ils représentent une donnéc de l'observation dont il n'y a aucune raison de sous-estimer l'intérêt au seul profit des formations superficielles du type grès à Sabals. Ils nous apportent la preuve que la surface des grès à Sabals, tectoniquement déformée, a été largement retouchée et réincorporée dans un modelé polygénique dont les traits les plus récents ne sont pas les moins dignes d'intérêt.

\section{Conclusions :}

a) Sur le plan stratigraphique. - La sćrie paléozö̈que de la Montagne Noire débute comme la série « pourprée » bretonne, tantôt par un poudingue à galets de grès et de faciès « Montfort » - ce sont les poudingues du Cap de la Chèvre - tantôt, et on peut dire beaucoup plus généralement, par un poudingue à galets de quartz et de faciès «Gourin ». Nous considérons que ces deux poudingues représentent la base de la série ordovicienne, stratigraphiquement discordante sur les schistes briovẻriens.

Nous avons, en outre, suggéré une équivalence entre le poudingue à galets de quartz de la Montagne Noire et le « poudingue de Gourin » de la carte géologique ( $2^{\circ}$ édition). Le fait de contester cette assimilation ne saurait, en aucune manière, porter atteinte au bien-fondé de la première conclusion qui, à nos yeux, demeure essentielle, puisque nous voyons dans le poudingue de la Montagne le signe concret d'une coupure stratigraphique fondamentale entre la série paléozoïque et le $\mathrm{X}$.

b) Sur le plan tectonique nous reconnaissons que la part faite à l'hypothèse demeure très large car la région fournit fort peu d'éléments d'appréciation. Mais nous pensons que le schéma proposé échappe à certaines contradictions associées au schéma clas- 
sique. Le témoignage des cartes auquel nous avons pris soin de nous référer constamment est d'ailleurs sa plus authentíque justification. Enfin nous avons pris soin de ne pas dissocier, sur le terrain, l'étude des données de la stratigraphie - voire de la sédimentologie - de celles de la tectonique. L'esquisse ici proposée est donc une synthèse provisoire des conclusions auxquelles nous avons abouti. Avant d'examiner les incidences de la tectonique du socle armoricain sur la couverture sédimentaire de sa bordure orientale il nous a paru nécessaire de chercher à définir l'allure du dispositif structural hérité, dans les portions de ce socle accessibles à l'observation directe.

c) Sur le plan morphologique, enfin, nous pensons avoir établi que le versant Sud de la Montagne Noire doit être considéré comme un talus structural, ouvre commune de la tectonique et de l'érosion différentielle, et nous estimons que le glacis de piémont au-dessus duquel il s'enlève n'est pas la surface des grès à Sabals, car il n'existe, au voisinage de la Montagne, aucun témoin incontestablement en place de la croûte siliceuse éogène.

Mais nous avons admis que, dans son ensemble, le glacis cornouaillais parait dériver de cette surface, tout en observant que la présence de galets roulés et de poudingues à ciment ferrugineux, beaucoup plus récents, nous invitait à ne pas sous-estimer la part des épisodes pliocènes dans l'élaboration des formes du modelé.

Note ajoutée à l'impression. - Nous avons pris connaissance, après la rédaction de cet article, d'une série de notes récentes de M. G. Mathieu (6) dans lesquelles l'auteur fait état, en ce qui concerne le massif vendéen, de considérations tectoniques proches de celles que nous venons d'exposer : interférence de phases orogéniques successives et superposition de trames directionnelles avec bandes mylonitisées jalonnant les lignes d'affrontement principales $(1 a, 1 b$ et surtout $1 c)$.

(6) G. MathiEU. - a) Les liaisons tectoniques entre Choletais et Bocage vendéen. Bull. Serv. de la Carte Géol. Fr., no 241, t. LII, 1954, p. 99-116.

b) Constitution géologique de la région de Cholet d'après la deuxième édition de la carte au $80.000^{\circ}$. C.R. Ac. Sc., t. CCXLI, p. 1216-1218, 31-10-1955.

c) Utilisation des amphibolites pour interpréter la tectonique du Bocage vendèen. C.R. Ac. Sc., t. CCXLIV, p. 635-637, 28-1-1957.

d) Observations géologiques sur le batholite de granite de Pouzauges (Vendèe). C.R. Ac. Sc., t. CCXLIV, p. 2625-2627, 20-5-1957 (écrit en collab. avec A. Brillanceau).

e) La bifurcation de l'anticlinal des Cornouailles dans le département des Deux-Sèvres. C.R. Ac. Sc., t. CCXLV, p. 705-707, 5-8-1957. 


\section{- $36-$ \\ REFERENCES BIBLIOGRAPHIQUES}

1. Raknors (Ch.) et Pruvost (P.). - Relations stratigraphiques des couches cambriennes de la Bretagne et du Maine. Ann. Soc. Géol. Nord, t. LVI, 1931.

1b. Pnecost (13.). .... A propos des poudingues briovériens el cambriens de Normandie et de Bretagne. C.R. Som. Soc. Géol. Fr., 19-6-1950, p. 205. 206.

2. Birot (A.). - Observations sur l'existence d'une série compréhensive dans le Massif armoricain. C.R. Som. Soc. Géol. Fr., 11-6-192:\}, p. $117-119$.

3. Bochnise (.J.-M.) el Gunchen (A.). - Les depots cocènes et leur signification morphologique en Bretagne méridionale et dans le Massii vendéen (note préliminaire). Bull. A.G.F., n 117 , déc. 1938, p. 136139.

4. Cogne (J.). -... Sur le complexe cristallin de la partie occidentale des Landes de Lanvaux. C.R. Ac. Sc., t. CCXXXIIl, 1951, p. 1375-1377.

5. Cowse (J.). - Schistes cristallins et granites en Bretagne méridionale L'anticlinal de Cornouaille. Bull. Soc. Géol. Fr., 6, III, 1953, p. 785808

6. Cogne (J.). -- Schistes cristallins el granites en Bretaune méridionale. A propos des hälle flints. Bull. Soc. Géol. Fr., 6, IV, 1954, p. 729-737.

7. Drusy (A.). - Sur le métamorphisme régional du Paléozoïque dans lanticlinal de Lanvaux, en Armorique méridionale et sur l'âge du grante de Lanvaux. C.R. Ac. Sc., t. CCXXXIII, 1951, p. 1636-1638.

8. Ijofnexox. - Sur l'âge et la composition des terrains de transition de l'Ouest de la France. Annales des Mines, 3, XIV, 1838, p. 213-258 el $351-398$.

9. Fatre-Mi:ret (MHe A.). - Lanticlinal de Lanvaux. Bull. Soc. (iéol. Fr., 5, XIV, 1944, p. 279-306.

10. Gior (P.-R.). - Sur le Briovérien au S. des Montagnes Noires. C.R. Somm. S. G.F., 18-12-1944, p. 179-181.

11. (:rot (P..R.). - Sur la lectonique des Montagnes Noires. C.R. Somm. S. G. F., 19-2-1945, p. 43-44.

12. Grot (P.-R.). - Essai synthétique sur la gréologie de la Cornouaille occidentale. Bull. Soc. Géol. Fr., j, XVIII, 1948, p. 3-14.

13. Gurchris (A.). - Le Relief de la Bretagne méridionale de la baie de Douarnenez à la Vilaine. Thèse, 1948 .

14. Hénert (E.). - Phyllades de St-Lô et conglomérats pourprés dans le N.W. de la France. Bull. Soc. Géol. Fr., 3, XIV, 1886, p. 713.

15. KLFin (C.). - Tectonique de couverture et discordance tectonisce en Armorique. Bull. A.G.F., nos 263-264, 1957, p. 29-39.

16. lineis (C.). - Quelques caractères originaux du socle armoricain. Norois, n" 15,1957 , p. 305-332.

17. Martonne (Em. de). - La pénéplaine et les côtes bretonnes. Ann. Géo., t. XV, 1906, p. 213-236.

18. MiLon (Y.). - L'extension des formations sidérolithiques éocènes dans le centre de la Bretagne. C.R. Ac. Sc., no 194, 1932, p. 1360-1362.

19. Valdaux (C.). -- La Montagne Noire de Basse-Bretagne. Ann. Géo., t. XIX, 1910, p. 209-230.

20. Wegman. (E.). - Note sur quelques problèmes de la tectonique superposéc. C.K. Sor. Géol. de Finlande, $\mathrm{n}^{\circ}$ 20, Helsinki, 1947. 


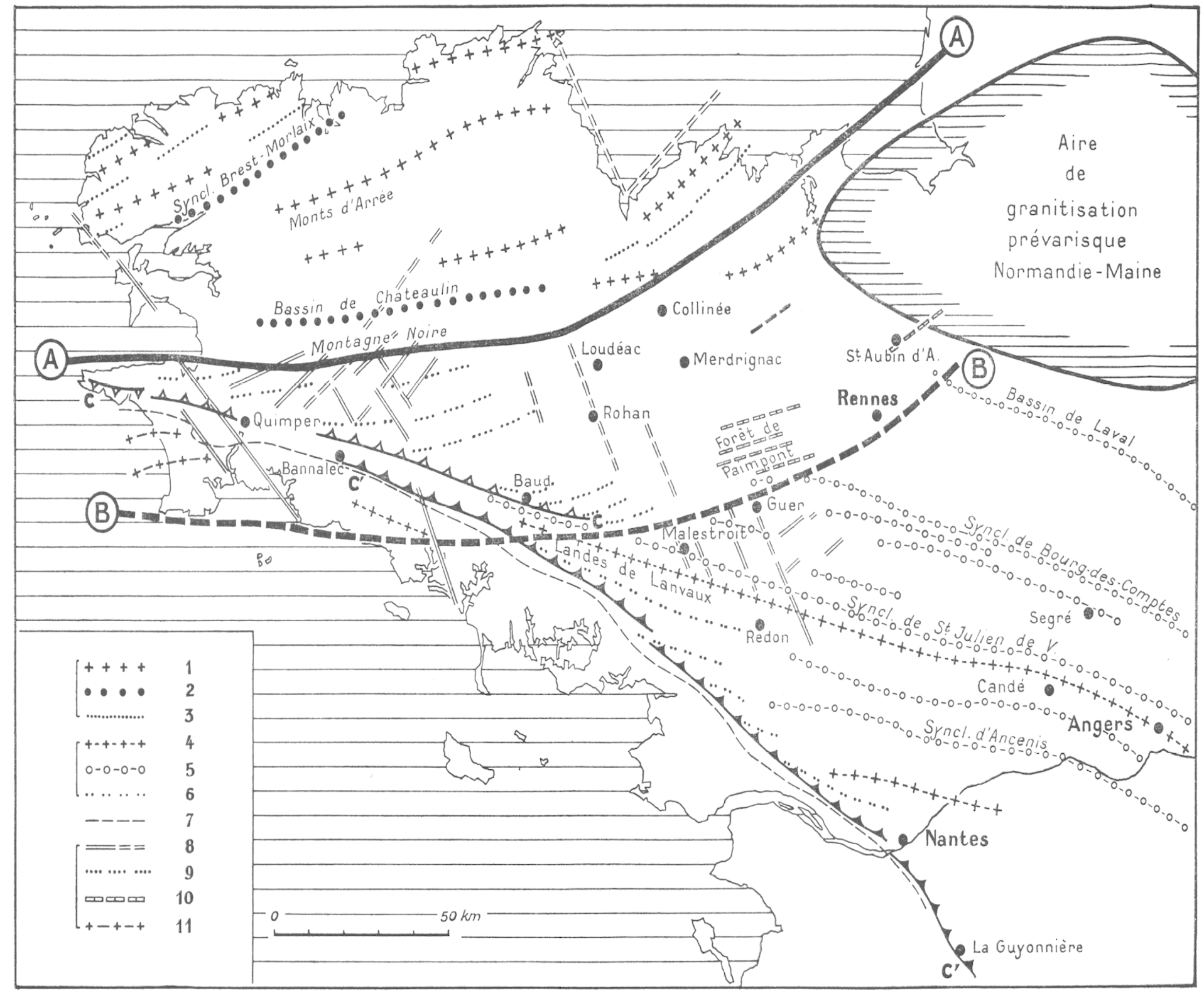

ESQLISSE TECTONIQCE des relations presumées entre la trame prévarisque et la trame hercynienne

A-A : llexure liminaire méridionale du bassin de Châteaulin et son prolonsement supposé (d'après la sravimétrie).

B-B : ligue :u S. de laquelle la direction «Lanvaux » parait prépondé-

C-C: bande mylonitisce septentrionale (Pte du Vian-Baud). Elle souligne une charniere lectonique à l'intersection des directions * Léon "

$C-C^{\prime}$ : bande mylonitisée méridionale (Bannalec-Nantes-la Guyonnière). \&. Lanvaux » avec le flanc Nord de "l'anticlinal de Cornouaille ».

I) $\mathrm{Au}$ Nord de $\mathrm{A}-\mathrm{A}$ (domaine de la direction « Léon $»$ ):

1. Axes des batholites de granite varisques. 3. Axes des trainées granulitiques varisques.

II) Au Sud et au S.E. de B-B (domaine où les directions $\mathrm{N}$. $100^{\circ} \mathrm{E}$. et

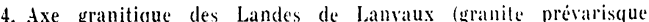
5. Axes des synclinaux siluriens situés de part et d'autre de t.

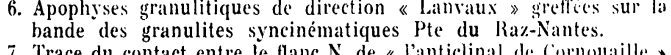
7. Trace du contact entre le flane N. de " T'anticlinal de Corrnoutille" et la hande granulitique Raz-Nantes.

III) Entre A-A et B-B : région dans laquelle la direction \& Léon » parait s'être superposée à une trame plus ancienne d'orientation «Lanvaux». 8. Failles ou axis de décrochement majeurs an long des flancs ș̣nclinaux en marge des régions I et II.

10. Axes tectonicues de la Forêt de Paimpont (escuisses do suncli-

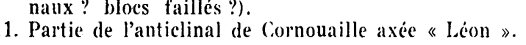

Yota (1) : C'est dans cette bande, puissamment tectonisée, qu'on observe - entre Collinée et St-Aubin-d’Aubigné - l'étranglement du "synclino-

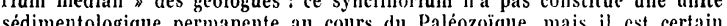
que les deux bassins subsidents de Châteaulin-Elorn à l'W. et de VitréLaval a l'E. ont largement communiqué au dévonien et au dinantien submergeant le seuil de Rohan-Loudéac.

Nota (2) : On observera que l'aire de granitisation prévarisque de Normandie-Maine parait avoir provoqué une sensible déflection de la direction

Erratum : ne pas tenir compte du signe 4 figuré au nord de Nantes. 